\title{
Aproximación a la determinación del déficit hídrico en la Región del Libertador General Bernardo O’Higgins, Chile, a partir de imágenes MODIS y datos meteorológicos ${ }^{1}$
}

\author{
Marcela Sánchez Martínez² y Luis Carvacho Bart ${ }^{3}$
}

\begin{abstract}
RESUMEN
Se comparan dos métodos para la determinación de déficit hídrico en la Región del Libertador General Bernardo O’Higgins. A partir de imágenes de satélite MODIS y datos meteorológicos, se calculan el Water Deficit Index (WDI) y el Temperature Vegetation Dryness Index (TVDI) cuyos insumos son temperatura de superficie, del aire e índices de vegetación y se aplican en el área de estudio. Se analizan los valores de los índices obtenidos para dos fechas específicas de primavera y verano y se determinan las semejanzas y diferencias espaciales y temporales entre ambos, tanto en magnitud de déficit hídrico o sequedad de suelo estimada como en tendencias observadas entre ellos, encontrándose solo pequeñas diferencias en los resultados según la estación del año. Finalmente, se concluye sobre las potencialidades y precauciones que se deben tener para hacer de estos índices herramientas útiles para la determinación del déficit hídrico del suelo.
\end{abstract}

Palabras clave: Déficit hídrico vegetal, índice WDI, índice TVDI, imágenes MODIS

\begin{abstract}
Two methods for the determination of hydric deficit in the Region del Libertador Bernardo O'Higgins, Chile are compared. Using land surface temperature and vegetation indices from MODIS satellite images and air temperature from meteorological records, the Water Deficit Index (WDI) and the Temperature Vegetation Dryness Index (TVDI) are calculated and applied in the study area. The values obtained for two specific dates in spring and summer are evaluated and spatial and temporal similarities and differences between them are measured both in magnitude of hydric deficit (or dryness) and in their tendencies. Regarding this, only small differences were detected. Finally, the potential and cautions in the use of these indices are discussed.
\end{abstract}

Key words: vegetal water deficit, WDI index, TVDI index, MODIS satellite images.

\footnotetext{
1 Proyecto FONDECYT 1085249. Artículo recibido el 10 de enero de 2012, aceptado el 30 de agosto de 2012 y corregido el 1 de octubre de 2012.
}

\footnotetext{
2 Instituto de Geografía, Pontificia Universidad Católica de Chile (Chile).E-mail: mism@uc.cl

3 Instituto de Geografía, Pontificia Universidad Católica de Chile (Chile). E-mail: Icarvach@uc.cl
} 
Los datos satelitales ofrecen considerables ventajas para el conocimiento de la variación espacial y seguimiento temporal de múltiples fenómenos de interés agronómico, hidrológico y muchos otros estudios geográficos y ambientales. Dentro de las variables que es posible obtener desde el espacio para este tipo de estudios están los índices de vegetación (IV) y la temperatura de superficie (TS). Uno de los IV más utilizados en el mundo es el Índice de Vegetación de Diferencia Normalizada, NDVI (Normalized Difference Vegetation Index), de fácil cálculo e interpretación y que es utilizado para el seguimiento y evaluación de la dinámica global de la vegetación terrestre. Se emplea para conocer y seguir los cambios estacionales en el vigor de las cubiertas vegetales, el efecto de fenómenos esporádicos, como pueden ser los provocados por El Niño, o procesos continuos, como el calentamiento terrestre (Chuvieco, 2010). Las series multitemporales de IV se emplean para realizar mapas de presencia de vegetación, pero también para informar la condición en que esa vegetación se encuentra (Wan et al., 2004). Por su parte, la temperatura de superficie constituye el principal indicador de la distribución de la energía disponible en la superficie de la Tierra, a través de su efecto en el flujo de calor latente y sensible a la atmósfera, flujo de calor sensible hacia el suelo y radiación hacia la atmósfera (Price, 1982 y 1990; Kustas \& Norman, 1996; Gupta et al., 1997), por ello es un parámetro clave en los procesos físicos a las diferentes escalas espaciales, locales, regionales y globales (Wan et al., 2004).

Dentro del campo específico que interesa para este estudio, la TS y los IV obtenidos a partir de imágenes satelitales son variables que han sido ampliamente utilizadas para elaborar indicadores de déficit o estrés hídrico en los vegetales e informar, por consiguiente, sobre las condiciones de humedad del suelo en determinados momentos. También se emplean para realizar el seguimiento de periodos de sequía cuando la falta de agua o estrés es persistente (Sandholt et al., 2002; Fensholt \& Sandholt, 2003; Wan et al., 2004; García et al., 2007; Pertovt et al., 2007).

Numerosos estudios demostraron desde hace ya más de dos decenios una clara relación negativa entre la TS y los IV, en especial el NDVI (Nemani \& Running, 1989; Nemani et al., 1993; Choudhury, 1994; Prosper-Laget et al., 1995; Gupta et al., 1997; Yang et al., 1997; Kalluri et al., 1998, entre otros). Esta alta correlación ha demostrado con el tiempo un claro grado de coincidencia considerando sensores variados, épocas del año distintas y diferentes localizaciones. Sintetiza una serie de conexiones entre procesos, entre ellos la evapotranspiración y el estrés hídrico (Sánchez, 2002). En cubiertas vegetales la disminución en la TS a medida que se incrementa la densidad de vegetación (mayor NDVI) es producto del enfriamiento provocado en las plantas por el flujo de calor latente o evapotranspiración (ET). La pendiente de la línea de ajuste TS/NDVI se puede interpretar como un índice de la resistencia superficial a la evaporación o a la evapotranspiración, según se trate de suelo desnudo o cubierto parcial o totalmente de vegetación. Conforme con esto, ante condiciones meteorológicas similares, las diferencias en cuanto a esta resistencia entre fechas distintas se deben, principalmente, a las variaciones en la disponibilidad de humedad del suelo, lo que sugiere que la pendiente de la recta es similar al factor de disponibilidad de humedad (cuociente entre evapotranspiración real, ETR, y potencial, ETP). Tal como señala Goetz (1997), y como se ha comprobado en muchos estudios, la TS puede aumentar rápidamente ante condiciones de falta de agua o estrés hídrico.

La idea del uso de información térmica y algunas características de la vegetación para estudiar el estrés hídrico del dosel vegetal fue originalmente propuesta por Jackson et al. en 1977 al elaborar el denominado Índice de Estrés Hídrico del Cultivo, Crop Water Stress Index, CWSI (Fensholt \& Sandholt, 2003). Luego, numerosos estudios han seguido sugiriendo que el uso combinado de la TS y el NDVI pueden proporcionar información sobre el estrés vegetal y las condiciones de humedad de la superficie (Fensholt \& Sandholt, 2003). Estos métodos o modelos se derivan a partir de la representación gráfica de la dispersión entre la TS y un IV, normalmente el NDVI. Si en el área se encuentra una mayor diversidad de vegetación y de contenidos de humedad en el suelo, en algunos casos esta dispersión presenta una forma triangular y en otras condiciones un espacio trapezoidal. Los modelos derivados de esta relación inicial 
han sido aplicados en una gran variedad de tipos de vegetación y cultivos, de condiciones climáticas y a diferentes escalas espaciales, desde metros a globales (Sandholt et al., 2002; Wan et al., 2004; Vásquez et al., 2008).

El CWSI se ha empleado para detectar el estrés hídrico vegetal en climas áridos, semiáridos y también en cálido húmedos donde se requiere riego en la época seca. Esta información junto a un programa de riego adecuado es necesaria para maximizar los rendimientos agrícolas con un manejo eficiente del riego. En este sentido, una discusión sobre el método y una evaluación sobre su utilidad práctica en la calendarización y optimización del agua de riego en los cultivos agrícolas puede verse en López et al. (2009). Por otra parte, una de sus características es que solo es aplicable a superficies totalmente cubiertas por vegetación, lo cual limita su uso en el contexto del estrés hídrico y de la evapotranspiración para la mayor parte de las superficies, constituidas por un compuesto de suelo y vegetación (Morán et al., 1994; Vidal y Devaux-Ros, 1995). Morán et al. desarrollaron en 1994 un método gráfico que proporciona estimaciones de ETR muy precisas y déficit hídrico relativo en superficies compuestas. El método se basa en la representación gráfica de la fracción de cubierta vegetal, $f_{c}$ y la diferencia entre temperatura superficial y temperatura del aire, TS-TA, medidas en cada pixel, a diferencia del CWSI de Jackson et al. que considera solo la TS. La dispersión de puntos tiende a formar un trapecio como el que se aprecia en la Figura $\mathrm{N}^{0} 1$, en el que se relacionan valores de fracción vegetal y TSTA, dando lugar al Vegetation Index/Temperature Trapezoid, VITT.

En la Figura $\mathrm{N}^{\circ} 1$ los vértices superiores corresponden a altos valores de NDVI y representan cubiertas vegetales bien abastecidas de agua en el extremo izquierdo (punto 1) y cubiertas vegetales sometidas a estrés hídrico en el sector derecho (punto 2); mientras, los vértices inferiores indican suelos desnudos húmedos en el extremo inferior izquierdo (punto 3) y suelos secos en el inferior derecho (punto 4). Considerando que, normalmente, la TS es mayor a la TA registrada en las estaciones meteorológicas, una menor diferencia entre las temperaturas, hacia el límite izquierdo o húmedo del trapezoide, de-
Figura $\mathrm{N}^{\mathrm{o}} 1$

Representación teórica del Vegetation Index/ Temperature Trapezoid, VITT

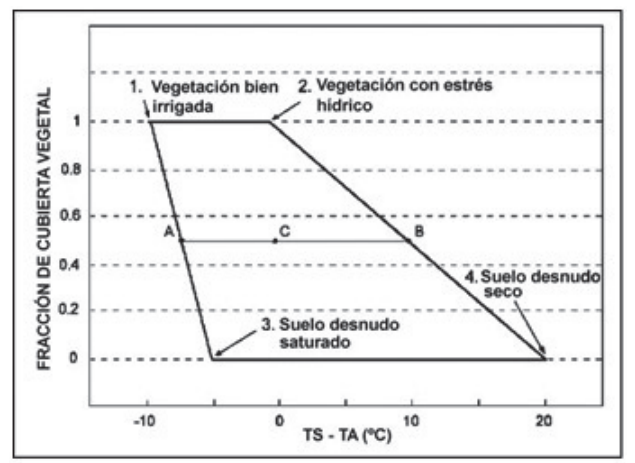

Fuente: Morán et al. (1994).

bería ser consecuencia del enfriamiento que supone la liberación de calor latente o ET que tiene lugar en la cubierta vegetal bien provista de agua; lo contrario ocurre cuando el suelo y la vegetación que sustenta no dispone de agua, la TS-TA se hace mayor y los píxeles tienden a situarse en el límite seco o derecho del trapecio. A partir de la figura que se genera en el VITT se puede calcular la razón entre el segmento $C B$, que representa la evapotranspiración real, y el segmento $A B$, correspondiente a la evapotranspiración potencial (Morán et al., 2004). En general, esta relación ETR/ETP se puede entender biofísicamente como un indicador de la disponibilidad de humedad en el suelo. En los términos en que lo plantean Morán et al., el complemento de esta relación sería el denominado Índice de Déficit Hídrico, Water Deficit Index (WDI), expresado como: WDI=1-ETR/ETP, el cual informa, como se dijo, del déficit hídrico relativo en suelos parcial o totalmente cubiertos de vegetación. El WDI oscila entre los valores 0 y 1 , indicando 0 nulo déficit hídrico y 1 déficit máximo. En términos aplicados la utilidad y el principal objetivo de este modelo radica en el hecho de que teniendo el dispersiograma, el cuociente entre las distancias CB/AB y conociendo la ETP a través de algún método convencional de precisión se puede obtener la ETR siguiendo la relación indicada, tal como señalan sus autores y confirman Yang et al. (1997) y el U.S. Water Conservation Laboratory (2001). En este sentido, se puede destacar la utilidad de las variables, TS, TA y 
NDVI, en la determinación directa de la ETR, conforme a diversos modelos, o en la interpolación espacial de la ETP obtenida con métodos convencionales (Sánchez y Chuvieco, 2002; Sánchez y Carvacho, 2006; Carvacho y Sánchez, 2008). Esto constituye un gran aporte de la percepción remota si estas medidas son lo suficientemente precisas, dada la dificultad de obtener una imagen continua de la ETP y la ETR por métodos convencionales de medida o, en su defecto, de estimación. Yang et al. (1997) confirman la utilidad del VITT en la evaluación de la disponibilidad de agua en el suelo y en la estimación de la evapotranspiración real en un área de Nueva Gales del Sur, Australia.

Por otra parte, y sobre la base de sus propios estudios y de otros autores, Gillies et al. (1997) utilizan la figura triangular que se forma en el dispersiograma TS/NDVI como indicativa del contenido de agua en el suelo y de la proporción de cobertura vegetal. En la Figura $\mathrm{N}^{\circ} 2$ se muestra un ejemplo de triángulo donde se señalan los valores de NDVI que indican una superficie cubierta totalmente por vegetación (NDVIs, vértice superior) y un suelo con los valores mínimos de NDVI en el área (NDVIo en la base del triángulo), en este caso suelo desnudo. El límite cálido de la figura estaría definido por los puntos que tienen el mínimo o nulo contenido de humedad para un mismo valor de NDVI y por ello tienen una TS mayor, y lo opuesto ocurre en los puntos próximos al límite frío que tendrían una máxima disponibilidad de agua.

Otros modelos que se asocian al espacio triangular que define la dispersión TS/NDVI son los propuestos por Wang et al. (2001) y el de Sandholt et al. (2002), entre otros. Wang, et al. (2001) establecen una aproximación para estudiar y realizar un seguimiento de sequías o periodos de estrés hídrico prolongado a escala regional, conocida como Vegetation Temperature Condition Index, VTCI. Wan et al. (2004) aplican este método en algunas zonas de los Estados Unidos de América utilizando composiciones de 16 días de datos del sensor Terra-MODIS. Como una forma de evaluar los resultados, los autores comparan los datos de NDVI, TS, TS/NDVI y VTCI con los datos de precipitaciones registradas en las mismas fechas de las composiciones y con los datos promedios de varios años con muy buenos resultados.
Figura $\mathrm{N}^{\circ} 2$

Gráfico de dispersión TS/NDVI para el 9 de agosto de 1990 en una cuenca de Arizona

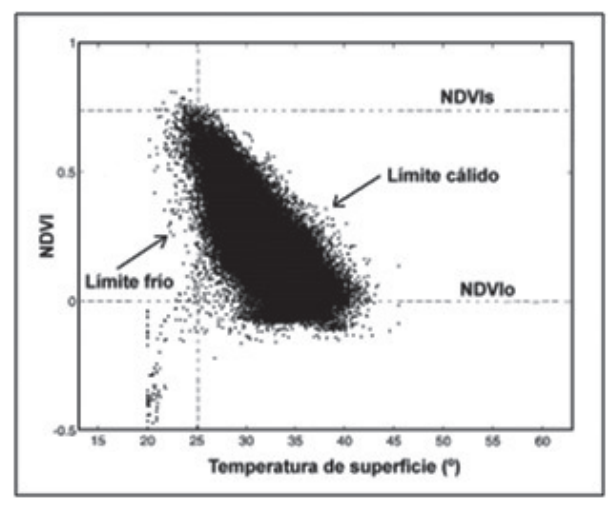

Fuente: Gillies et al. (1997).

El modelo de Sandholt et al. (2002), el Índice de Sequedad Temperatura-Vegetación, por su parte, es una simplificación empírica del WDI al que los autores denominan Temperature-Vegetation Dryness Index, TVDI. Fue desarrollado para establecer el estado de la humedad del suelo, aunque expresado en el término complementario de sequedad. Al igual que en los modelos gráficos WDI y VTCl, para calcular el índice es necesario realizar el dispersiograma correspondiente y definir los límites del espacio TS/NDVI para el área de estudio, definiendo el límite seco y el límite húmedo del triángulo para el área y momento de estudio. El modelo asume que la humedad del suelo es la principal fuente de variación de la TS y que el TVDI está relacionado a la humedad superficial del suelo debido a los cambios en la inercia térmica y al control de la evaporación y transpiración sobre la distribución de la radiación neta (balance de energía) (Sandholt et al., 2002). El modelo de esta representación y su significado físico-biológico general se observa en la Figura $\mathrm{N}^{0} 3$.

Establecidos los bordes o límites del triángulo es posible calcular el TVDI o índice de sequedad para cada píxel, conociendo su posición en el espacio a través de sus valores TS y NDVI, y considerando la proporción entre las líneas $A$ y $B$ que se muestran en la Figura $N^{0}$ 4. Esta relación $A / B$ es análoga a la de la 
Figura $\mathrm{N}^{0} 1$, en el caso del VITT y del índice WDI, donde $C B / A B$ representan de alguna manera una estimación de la disponibilidad de humedad en la vegetación o en el suelo o, expresado en las palabras de Morán et al. (2004), de la relación entre ETR/ETP o déficit hídrico. Las isolíneas de TVDI en la Figura $\mathrm{N}^{\circ}$ 4 indican que píxeles que se encuentran en distinta posición del triángulo pueden alcanzar el mismo valor de TVDI. El TVDI varía entre 0 y 1 , indicando 0 máxima humedad del suelo y 1 mínimo contenido de humedad, los cuales se asocian con los correspondientes niveles de sequedad en la vegetación.

Figura $\mathrm{N}^{\circ} 3$

Modelo de la relación TS/NDVI

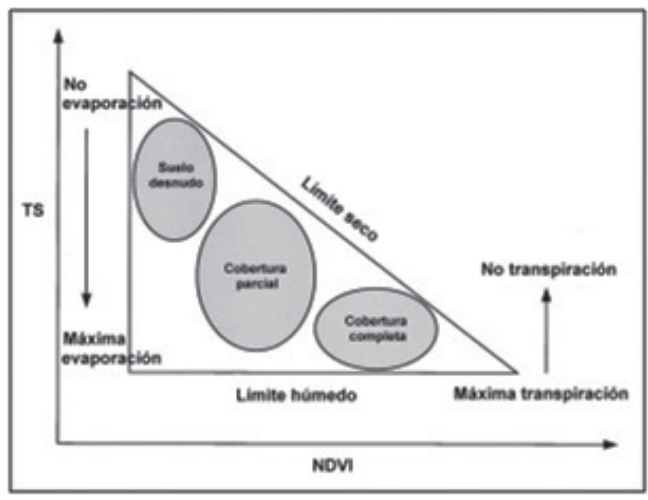

Fuente: Sandholt et al. (2002).

Figura $\mathrm{N}^{\circ} 4$

Triángulo que define los límites del TVDI

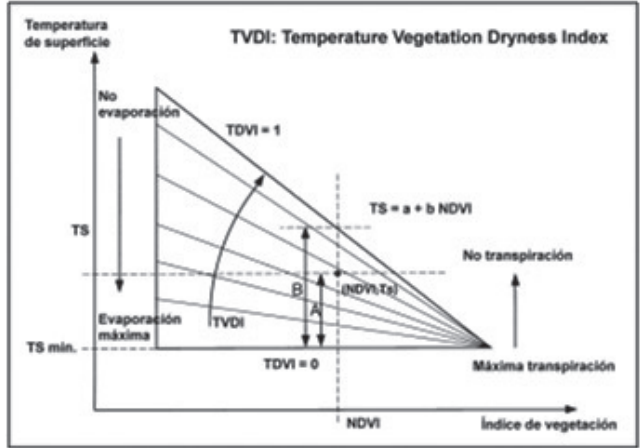

Fuente: Sandholt et al. (2002).

Aplicaciones del índice TVDI en Brasil han sido presentadas por Pertovt et al. (2007 y 2008). García et al. (2007), por su parte, realizan en la cuenca del río Segura, España, una comparación de los resultados obtenidos con el WDI, TVDI y VTCl, en una serie de imágenes MODIS de los años 2000 a 2006 concluyendo que los tres índices permiten detectar condiciones de escasez hídrica o de sequía, situaciones que no refleja el método basado en datos de precipitaciones que los autores aplican simultáneamente.

Tal como señalan Xin et al. (2006) en su propuesta aplicada en China, en los modelos gráficos presentados el límite frío o húmedo está definido por la mínima temperatura a distintos niveles de NDVI, es decir, que la humedad del suelo está a capacidad de campo o superior a ella y es suficiente para desarrollar el proceso de evaporación o transpiración, según el tipo de cubierta, suelo desnudo (mínimo NDVI) o suelo cubierto totalmente de vegetación (máximo NDVI). Opuestamente, el límite cálido o seco muestra la máxima TS para cada valor de NDVI y representa la condición de mayor sequedad dadas las circunstancias del momento.

Considerando las ventajas y los buenos resultados alcanzados en distintas regiones del mundo por los métodos presentados, este trabajo aplica los índices WDI y TVDI en la Región del Libertador General Bernardo O’Higgins, esperando obtener con el WDI una estimación del déficit hídrico de la cubierta del suelo y del estado de la humedad del suelo (o sequedad) con el TVDI. Ambos índices se encuentran conceptualmente relacionados, dado que se considera que el déficit hídrico está vinculado en forma directa con el contenido de humedad del suelo y, por tanto, con el vigor vegetal (NDVI) que, a través de la evapotranspiración, tiene su reflejo en las variaciones de la TS. Se espera analizar y comparar los resultados obtenidos con estos métodos que no se han empleado todavía en nuestro país.

\section{Área de estudio}

Corresponde a la Región del Libertador General Bernardo O’Higgins, localizada en Chile, directamente al sur de la Región Metropolitana de Santiago y de la Región de Valparaíso (Figura $N^{\circ} 5$ ). Se extiende, aproximadamente, entre los $33^{\circ} 50^{\prime}$ y los $34^{\circ} 50^{\prime}$ de latitud sur, y desde la cordillera de Los Andes en su límite con Argentina, por el este, hasta 
el océano Pacífico, por el oeste. Tiene una superficie de $16.387 \mathrm{~km}^{2}$ y en ella se presentan las cuatro grandes unidades morfológicas que caracterizan el territorio nacional sudamericano: de este a oeste la cordillera de los Andes, la depresión intermedia, la cordillera de la Costa y las planicies litorales. Ambas cordilleras se acercan en algunos sectores para conformar la cuenca de Rancagua en la depresión intermedia. La disposición general del relieve y la situación de la región con respecto al océano generan contrastes espaciales en los elementos naturales del paisaje, entre ellos, el clima, las características del suelo y la distribución de la vegetación, así como también en las actividades económicas de carácter silvoagropecuario. En la Región predomina el clima de tipo mediterráneo con Iluvias durante la estación fría y estación cálida seca; esta última alcanza entre cuatro y seis meses, según la estación meteorológica considerada (Novoa y Villaseca, 1989). En la alta cordillera se presenta un clima frío provocado fundamentalmente por la altura. Se distinguen claramente las cuatro estaciones del año, lo que influye con fuerza en la producción agrícola regional, actividad muy destacada en la región.
La red hidrográfica regional está representada principalmente por el río Rapel, formado por la confluencia de los ríos Cachapoal, por el norte, y Tinguiririca, por el sur, que nacen en el sector andino. El Rapel recibe en su curso inferior al estero Alhué, cuya cuenca es costera y tiene su origen en la Región Metropolitana de Santiago.

El período de estudio mayor del que forma parte el trabajo que se presenta corresponde al comprendido entre el 1 de enero de 2001 y el 31 de diciembre de 2006. Este periodo combinado con las características regionales generales, definen un área apta para acometer un estudio exploratorio de esta naturaleza.

\section{Metodología}

\section{Datos satelitales y meteorológicos}

Tal como se ha indicado, los índices WDI y TVDI se calculan a partir de la dispersión que muestran en el área de estudio los valores de las variables NDVI y la diferencia entre la TS y la TA, en el caso del WDI, y entre el

Figura $\mathrm{N}^{\circ} 5$

Región del Libertador General Bernardo O’Higgins

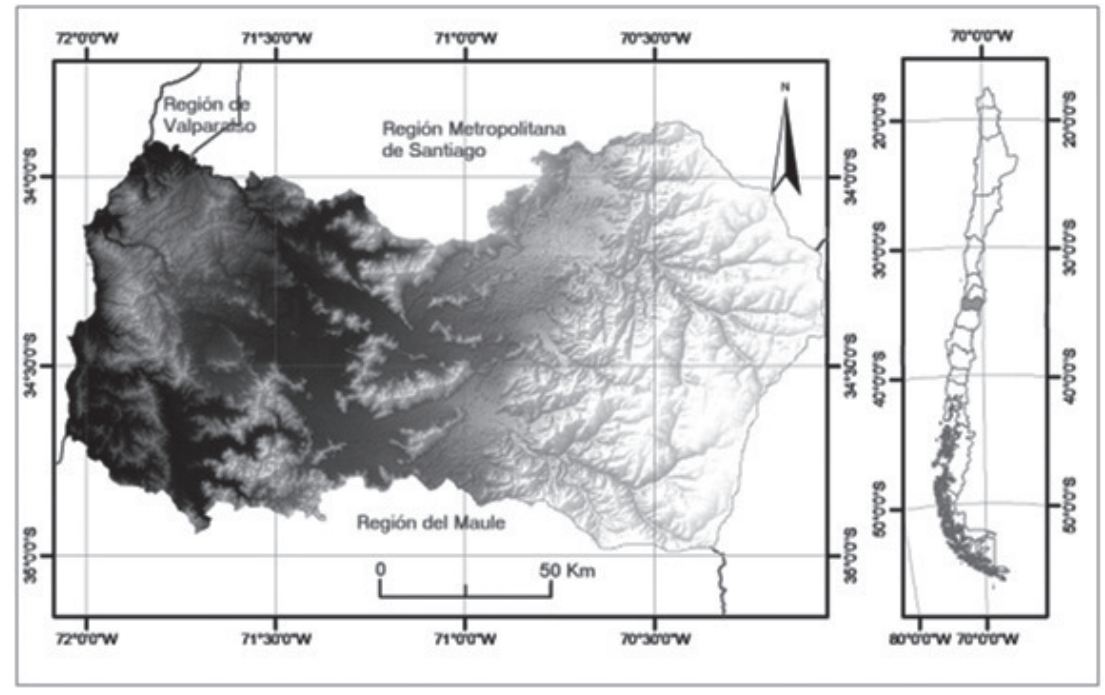

Fuente: Elaboración propia. 
NDVI y la TS para el TVDI. NDVI y TS han sido calculados en este estudio a partir de imágenes MODIS y la TA obtenida de datos registrados en estaciones agrometeorológicas convencionales.

Las imágenes MODIS fueron descargadas para el periodo 2001-2006 desde sitios FTP de NASA y luego procesadas mediante la herramienta HEG Tool provista por el mismo organismo. Para todas las imágenes descargadas que correspondían a horas de sol (entre las 12.00 horas y las 18.00 horas) se calculó el NDVI a 250 m de resolución espacial de acuerdo a la siguiente fórmula (Tucker, 1980):

Donde:

$N D V I=(N I R-R e d) /(N I R+R e d)$

NDVI :índice de vegetación de diferencia normalizada

NIR :valores de la banda de infrarrojo cercano

Red : valores de la banda del rojo

La TS, por su parte, de $1 \mathrm{~km}$ de resolución espacial, se calculó utilizando datos de radiancia de la imagen y los coeficientes de calibración respectivos presentes en los metadatos. Luego, la temperatura se calcula utilizando la ley de Planck (Anderson, 2005):

\section{Donde:}

$$
T=\left(C 2 * V_{C}\right) / \ln \left(1+C 1 * V C^{3} / \mathrm{Ne}\right)
$$

$T$ :temperatura en grados Kelvin

C1 :1,191062*10-5

C2 : 1,4387863

$\mathrm{Ne}$ : imagen de radiancia

$V_{C}$ : valor del centroide de cada banda (tomado de los metadatos de la imagen)

Las imágenes resultantes se convierten a grados Celsius (sustrayendo 273,5 a cada imagen de temperatura en Kelvin) y luego se remuestrean a $250 \mathrm{~m}$. Posteriormente, se aplicó a todas ellas una máscara para aislar la región de interés.

Se adquirieron los datos meteorológicos diarios de temperatura del aire y de precipitación de 15 estaciones pertenecientes a la red agrometeorológica administrada por la Dirección Meteorológica de Chile, distribuidas en la Región, y seis en las zonas próximas a ella de las regiones Metropolitana, de Valparaíso y del Maule, para el mismo periodo de estudio.
Estas estaciones están ubicadas en sectores agrícolas representativos de la zona.

\section{Aplicación de los índices de estrés} hídrico

El WDI y el TVDI pueden ser aplicados sobre imágenes diarias o sobre compuestos de varios días; en este caso se emplearon imágenes diarias de dos fechas de diferentes disponibilidades y requerimientos de agua dentro del año y en dos años distintos. Considerar cuatro fechas permitió tener en cuenta en la comparación una mayor variabilidad eventual del fenómeno. Las fechas seleccionadas son el 31 de octubre de 2003, el 31 de enero de 2004, el 31 de octubre de 2005 y el 24 de enero de 2006.

\section{Cálculo del Water Deficit Index, WDI}

El método propuesto por Morán et al. en 1994 fue aplicado según la siguiente relación que presentan Vidal y Devaux-Ros (1995) y Desbois et al. (1997):

$1-W D I=\frac{B C}{A B}=\frac{(T S-T A)-(T S-T A)_{d r y}}{(T S-T)_{w e t}-(T S-T A)_{d r y}}=\frac{E T R}{E T P}$

Donde:

WDI: Water Deficit Index

$B C$ y $A B$ : distancias representadas en el trapecio

$(T S-T A)$ : diferencia de temperaturas medida en el píxel

$(T S-T A)_{d r y}:$ diferencia de temperaturas en el límite derecho del trapecio

$(T S-T A)_{\text {wet }}$ : diferencia de temperaturas en el límite izquierdo del trapecio

ETR: evapotranspiración real

ETP: evapotranspiración potencial

Si bien la formulación original del método se aplica con el parámetro fracción de cobertura vegetal, $f_{C}$, varios estudios han demostrado que el VITT y el WDI se pueden aplicar utilizando índices de vegetación (linealmente relacionados con $f_{c}$ ) como el NDVI o el SAVI (Morán et al., 1994; Vidal y Devaux-Ros, 1995; Morán et al., 1996). En este trabajo se calcularon sobre imágenes de NDVI. Por otra parte, para calcular la imagen de diferencia TS-TA fue necesario obtener una imagen continua de la TA, para lo cual se realizó una 
interpolación espacial de la misma utilizando el método IDW (Inverse Distance Weighting) en las fechas seleccionadas. Dada la ausencia de datos puntuales de TA en la precordillera y cordillera de los Andes para realizar una adecuada interpolación espacial, y considerando que el interés del trabajo se centra más en las de cobertura vegetal de la depresión intermedia y la cordillera de la Costa, se generó una máscara a partir de un modelo digital de elevaciones que dejara fuera de análisis posterior a los píxeles situados a elevaciones superiores a $1.500 \mathrm{~m}$. Dicha máscara fue también remuestreada a $250 \mathrm{~m}$.

Teniendo el NDVI, TS y TA para cada una de las cuatro fechas indicadas se realizaron los dispersiogramas entre NDVI y TS-TA con todos los datos de la región. En cada uno se trazaron los límites del trapezoide, línea húmeda por el lado izquierdo y línea seca por el derecho. Estos extremos pueden ser determinados a partir de cálculos teóricos del balance de energía superficial, utilizando información proporcionada por satélites y por las estaciones meteorológicas convencionales, o bien en forma directa, a partir de los índices de vegetación y de las diferencias de temperaturas (Vidal y Devaux-Ros, 1995), alcanzando ambos métodos un muy buen grado de ajuste cuando en los datos derivados de las imágenes existe una amplia variabilidad. En este caso se realizó de manera directa utilizando las observaciones de máximo valor de NDVI para cada observación de TS-TA, con lo que basta con seleccionar solo dos pares característicos de coordenadas TS-TA/NDVI para determinar la ecuación de la línea seca. Un proceso análogo se utiliza para determinar la línea de límite húmedo, pero eligiendo esta vez los mínimos valores de NDVI para cada observación de TS-TA.

Se creó un módulo en el SIG ArcGIS cuyos insumos eran solamente las coordenadas de inicio y término de los límites seco y húmedo y las imágenes de TS-TA y NDVI para obtener imágenes que permitieran conocer para cada píxel de la región su posición dentro del gráfico y la distancia hacia los límites izquierdo y derecho (relación $\mathrm{CB} / \mathrm{AB}$ en la Figura $N^{0} 1$ ) y de cuya relación fue posible, finalmente, calcular el WDI. Se obtuvieron así las cuatro imágenes del WDI.

\section{Cálculo del Temperature-Vegetation Dryness Index, TVDI}

Según sus autores, Sandholt et al. (2002), el modelo se formula como:

$$
T V D I=\frac{T S-T S_{\min }}{(a+b \times N D V I)-T S_{\min }}
$$

Donde:

TVDI : Temperature-Vegetation Dryness Index. $T S_{\min }$ : TS mínima en el triángulo y que define el límite húmedo.

TS : TS observada en el píxel.

NDVI :NDVI observado en un píxel determinado.

$a$ y $b$ : parámetros que definen el límite seco del triángulo modelado como el ajuste lineal de los datos $T S_{\text {máx }}=a+b x N D V I$, donde $T S_{\text {máx }}$ es la $T S$ máxima observada para un NDVI determinado. $a$ y $b$ pueden ser estimados en la imagen.

Para la aplicación de este índice debió realizarse para cada fecha el diagrama de dispersión TS/NDVI como punto de partida y a continuación definir los límites seco y húmedo de la figura triangular que tiende a formarse en la dispersión. El borde seco fue trazado, tal como señalan sus autores, a través de la recta de ajuste de los puntos que corresponden a los de mayor TS para cada nivel de NDVI. Para realizar esto se usó un procedimiento análogo al utilizado para definir los límites del índice WDI ya descrito anteriormente, como asimismo para el trazado del límite húmedo. Establecidos los límites solo resta conocer la posición de cada píxel del área estudiada dentro de ellos y calcular la relación $A / B$ que se muestra en la Figura $N^{\circ} 4$, para lo cual se desarrolló también un módulo en ArcGIS que utilizando solo las coordenadas que definen los límites seco y húmedo y las imágenes de TS y NDVI crea los mapas de TVDI.

Cabe agregar a lo antes dicho que se eliminó el área correspondiente a la precordillera y cordillera de los Andes sobre los 1.500 metros dado que su inclusión tienden a distorsionar los límites de los polígonos y con ello los valores de los índices si se consideran sus datos de TS. Ello debió hacerse debido a que en esa 
zona se registran bajas temperaturas todo el año, especialmente en el invierno, producto de los efectos propios del relieve y la altitud sobre ella y otros elementos del clima, no siendo respuesta al fenómeno físico-biológico de la evapotranspiración que es lo que sí interesa recoger y considerar en el modelo.

Como una manera de explicar, al menos parcialmente, la variación espacial de los índices se realizó un análisis de correlación entre ellos y los datos de precipitaciones registradas en las estaciones meteorológicas de la región. Se consideraron las precipitaciones totales registradas en el mes en que se calculan los índices, las del mes anterior y la acumulación de ambos meses. Como referencias del uso de datos de precipitaciones en estudios de este tipo, sea con fines de validación o explicación, están Vicente-Serrano et al. (2004), Wan et al. (2004) y García et al. (2007).

\section{El déficit hídrico de la Región del Libertador General Bernardo O’Higgins}

En la Figura $N^{\circ} 6$ se muestran como ejemplo del trabajo realizado las imágenes de
NDVI, TS, TA y TS-TA obtenidas para el día 31 de octubre de 2003. En ellas se pueden apreciar las diferencias espaciales que se producen en las variables consideradas, según las distintas localizaciones en la región. Resultados análogos se obtienen en las otras tres fechas estudiadas.

El comportamiento general de las variables (Figura $\mathrm{N}^{\circ}$ 6) refleja muy bien las principales características geomorfológicas y climáticas de la región y, consecuentemente, las asociadas a la cubierta del suelo. NDVI: es muy variable, dándose valores que oscilan entre $-0,24$ y 0,85 , aunque esos valores extremos corresponden a píxeles aislados. Se aprecia con claridad el límite entre el secano costero y el secano interior. Los menores valores se presentan en la alta cordillera andina, desprovista de vegetación. TS: en este día varía entre los -4 y $47^{\circ} \mathrm{C}$. Los mayores valores se presentan en el sector norte de la región, próximos al lago Rapel, y los más bajos como es de esperar en la cordillera andina. TA: la falta de datos meteorológicos no permite obtener una mejor representación de algunas áreas; no obstante, es posible apreciar señales de cierta consistencia con los datos de TS, ya que los mayores valores de

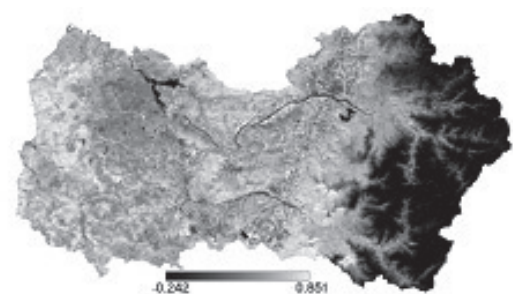

NDVI

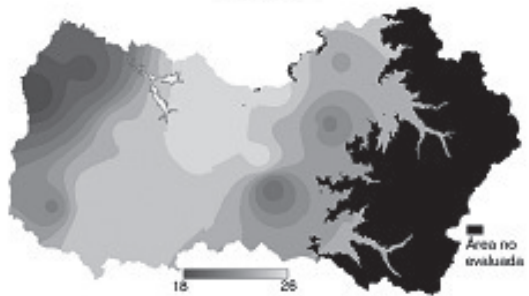

TA

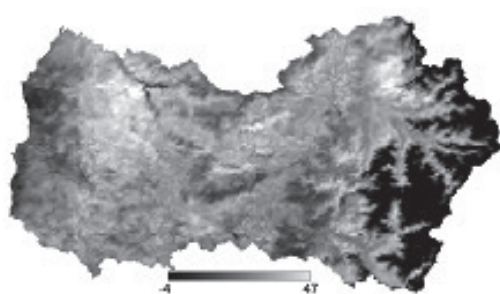

TS

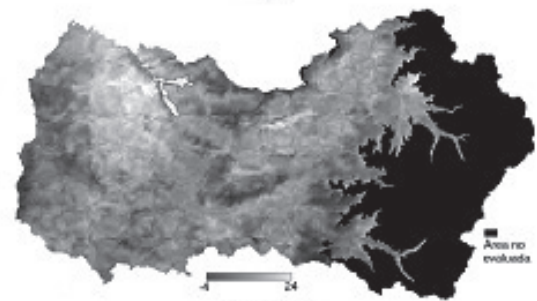

TS-TA

Fuente: Elaboración propia. 
TA en este día se registran en algunas partes de la depresión intermedia y sobre todo en el norte de la región, hacia el sector del secano costero. Aquí se encuentra la zona más cálida de la región, representada por la estación La Rosa, Marchigüe, Colchagua y San Vicente (Dirección Meteorológica de Chile, 2003). En la costa norte sobresale Tanumé con su baja TA característica. TS-TA: también muestra contrastes espaciales en la región, conforme a las variables que le dieron origen, espe- cialmente la TS, dada la mayor variabilidad respecto a la TA interpolada.

A partir de las variables básicas mostradas se realizaron los gráficos de dispersión procedentes y se trazaron los trapezoides correspondientes, a partir de los cuales se calculó el WDI, expresados en valores entre 0 y 1 . Se obtuvieron las ocho imágenes esperadas que presenta la Figura $N^{0} 7$.

Figura $\mathrm{N}^{\circ} 7$

Water deficit index, WDI y TVDI en las fechas seleccionadas

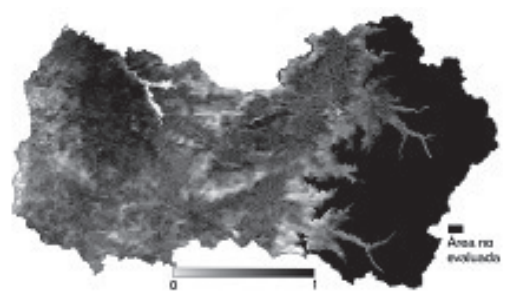

WDI $31 / 10 / 2003$

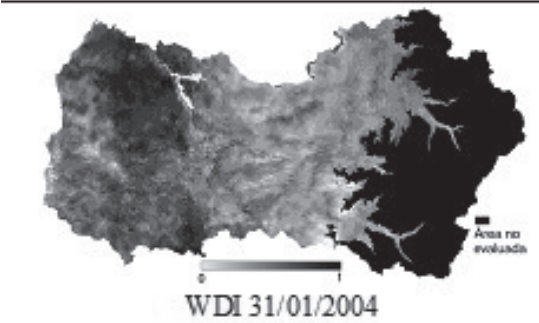

WDI $31 / 01 / 2004$

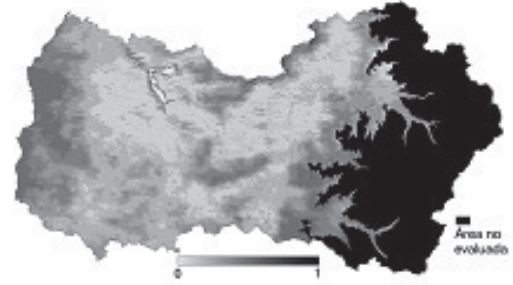

WDI $31 / 10 / 2005$

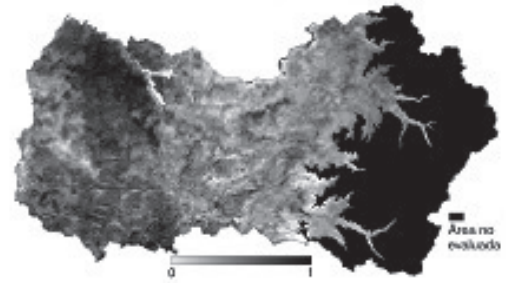

WDI 24/01/2006

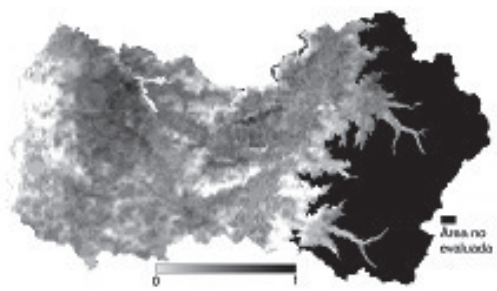

TVDI $31 / 10 / 2003$

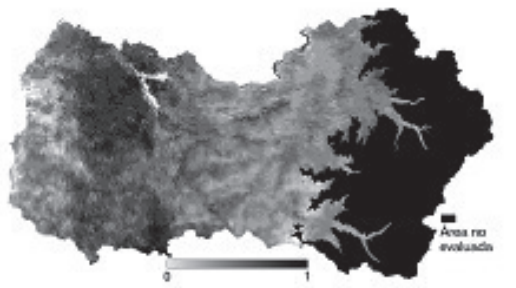

TVDI $31 / 01 / 2004$

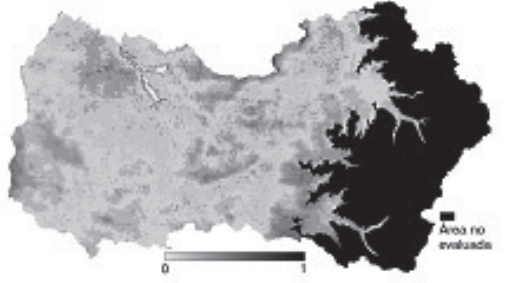

TVDI 31/10/2005

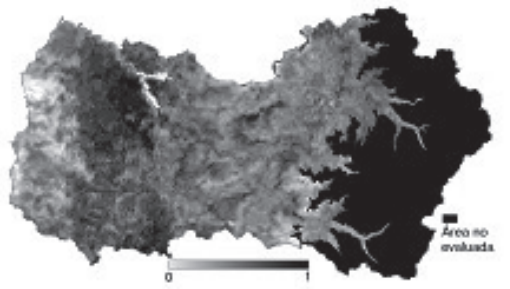

TVDI $24 / 01 / 2006$

Fuente: Elaboración propia. 
En la Figura $\mathrm{N}^{0} 7$ se aprecia la consistencia en la distribución de las áreas de menor déficit hídrico o sequedad (más claro) y las de mayor déficit hídrico o sequedad (más oscuro) entre ambos índices. Se advierte también la gran variabilidad de los valores de WDI y TVDI en la región obtenidos en las cuatro fechas, lo cual reproduce las grandes diferencias naturales y también antrópicas (uso/ cubierta del suelo) que se presentan en la región. Evidentemente, y teniendo en cuenta las particularidades propias de cada fecha y año considerado, los índices son menores en las imágenes de octubre en comparación a los de enero y en las dos temporadas consideradas, es decir, octubre presenta un menor déficit hídrico o menor sequedad que enero.

En todas las imágenes se advierte muy claramente la diferencia entre el sector del secano costero, más húmedo, y del secano interior y depresión intermedia, más secos, reflejando la influencia oceánica en la región.
Las zonas precordilleranas y otras de mayor altura presentan menores valores dada su cubierta vegetal, más vigorosa después del invierno. Considerando una variación estacional dentro del año, mayor contraste es posible ver entre el 31 de octubre de 2005 y el 31 de enero de 2006 que en las imágenes ejemplos de la temporada anterior 2003-2004, lo que indica la sensibilidad de los índices para recoger y representar situaciones distintas a lo largo del año y entre años. Ciertamente, hace falta considerar una secuencia estacional más completa para obtener resultados más contundentes a la luz de lo promisorio que se han mostrado los obtenidos hasta el momento. Más allá del estudio de episodios de déficit o estrés hídrico, el estudio de una serie temporal de varios años podría colaborar en la detección de eventuales situaciones de sequías prolongadas, comprender su desarrollo espacial en la región y ayudar a encontrar soluciones que mitiguen esos efectos ante sucesos futuros.

Figura $\mathrm{N}^{\circ} 8$

Relación entre el WDI y el TVDI en las fechas seleccionadas
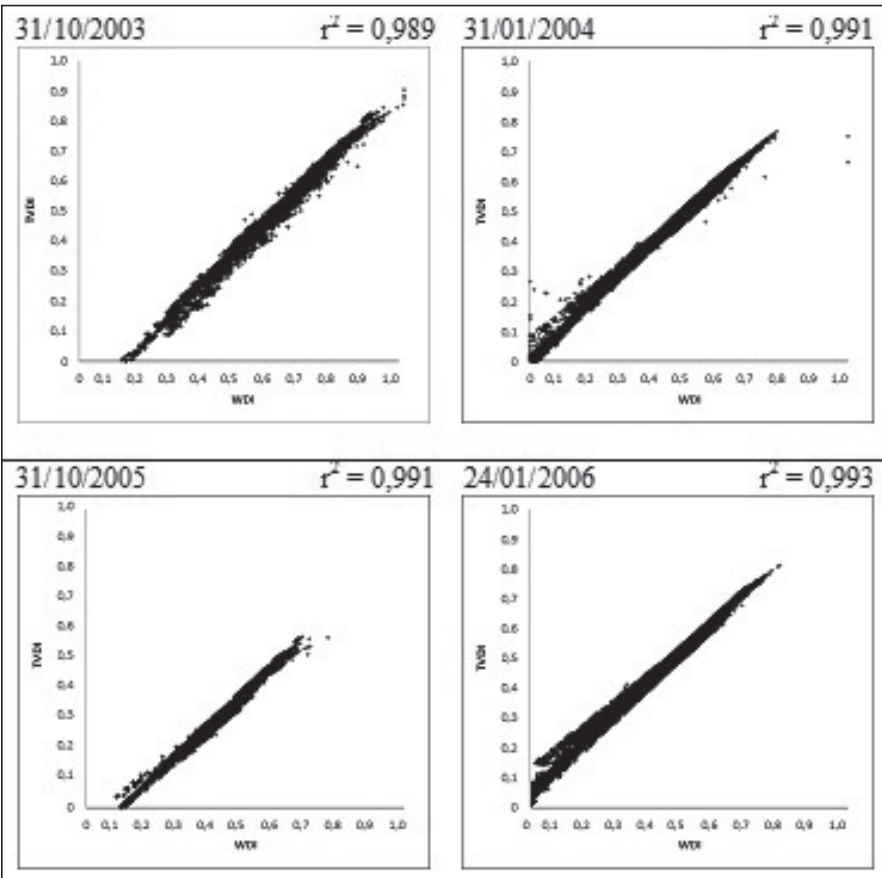

Fuente: Elaboración propia. 
La similitud entre los resultados obtenidos con WDI y TVDI pueden ser evaluados de dos maneras, a través de la semejanza de la tendencia de las observaciones o por medio de la semejanza de las observaciones mismas. En este caso, por una parte, la similitud de la tendencia es muy alta en todas las fechas estudiadas donde se obtiene un coeficiente de determinación superior a 0,989 (no se incluyen las áreas andinas sobre los $1.500 \mathrm{~m}) \mathrm{y}$, por otro lado, las magnitudes de los valores presentan diferencias claramente observables en los gráficos de dispersión que se muestran en la Figura $N^{\circ}$ 8. El índice de déficit WDI entrega valores mayores que los del índice de sequedad TVDI para los dos días de octubre considerados en este estudio. En los días de enero, los valores de WDI y TVDI son muy parecidos. Estas situaciones muestran que en ciertas circunstancias los índices reaccionan con distinta sensibilidad a las variables que determinan el fenómeno de disponibilidad de agua en el suelo y que se reflejan en el vigor vegetal y en la temperatura superficial y del aire.

La última parte de los resultados se refieren al análisis de correlación entre los índices y las precipitaciones registradas en las estaciones meteorológicas, al respecto se puede decir que, aunque la correlación es, en general, negativa como se esperaba, a mayor precipitación menores valores de déficit o sequedad, los resultados del análisis no son consistentes en todas las fechas.

En el cuadro $N^{\circ} 1$ se muestran los valores $r$ obtenidos en las cuatro fechas consideradas. En enero no se aprecian valores de $r$ consistentes con lo esperable, dado que no se producen precipitaciones o son muy bajas y debido a que el riego incorpora humedad al suelo que no está siendo considerado en los montos pluviométricos. Para octubre los mayores coeficientes de correlación se presentan entre la precipitación del mes de octubre de 2003 con el valor del TVDI del día 31 de octubre $(r=-0,78)$ y entre las precipitaciones acumuladas de septiembre y octubre con el TVDI de 2005 y 2003 ( $r=-0,69$ y $r=-0,64$, respectivamente). Estos resultados, si bien se aproximan en algunos casos a los observados por Wan et al. (2004), permiten deducir que el comportamiento de los índices no solo obedece al de las precipitaciones registradas, debiéndose considerar otros factores en su explicación, tal como sugieren VicenteSerrano et al. (2004) y Pertovt et al. (2008). También es posible, como se ha hecho en otras investigaciones, trabajar con índices de sequía basados en datos meteorológicos más elaborados y bien ajustados a mediciones del contenido de humedad del suelo como una manera de evaluar los resultados obtenidos. Entre ellos está el Índice de Precipitación Estandarizada, Standardized Precipitation

Cuadro $\mathrm{N}^{\circ} 1$

Coeficientes de correlación entre las precipitaciones y los índices WDI y TDVI ( $\mathrm{n}=15)$

\begin{tabular}{|l|r|r|c|r|r|}
\hline $\begin{array}{c}\text { Precipitación } \\
\text { mensual }\end{array}$ & $\begin{array}{c}\text { WDI } \\
31 / 10 / 03\end{array}$ & $\begin{array}{c}\text { TVDI } \\
31 / 10 / 03\end{array}$ & Precipitación mensual & $\begin{array}{c}\text { WDI } \\
31 / 01 / 04\end{array}$ & $\begin{array}{c}\text { TVDI } \\
31 / 01 / 04\end{array}$ \\
\hline Oct. 2003 & $-0,45$ & $-0,78$ & Ene. 2004 & - & - \\
\hline Sept. 2003 & $-0,30$ & $-0,32$ & Dic. 2003 & 0,19 & 0,36 \\
\hline Sept.-Oct. 2003 & $-0,46$ & $-0,68$ & Dic.2003-Ene.2004 & 0,19 & 0,36 \\
\hline $\begin{array}{l}\text { Precipitación } \\
\text { mensual }\end{array}$ & $\begin{array}{r}\text { WDI } \\
\text { W1/10/05 }\end{array}$ & $\begin{array}{r}\text { TVDI } \\
31 / 10 / 05\end{array}$ & Precipitación mensual & $\begin{array}{r}\text { WDI } \\
24 / 01 / 06\end{array}$ & $\begin{array}{r}\text { TVDI } \\
24 / 01 / 06\end{array}$ \\
\hline Oct. 2005 & $-0,24$ & $-0,32$ & Ene. 2006 & - & - \\
\hline Sept. 2005 & $-0,47$ & $-0,66$ & Dic. 2005 & 0,36 & $-0,05$ \\
\hline Sept.-Oct. 2005 & $-0,51$ & $-0,69$ & Dic.2005-Ene.2006 & 0,36 & $-0,05$ \\
\hline
\end{tabular}

Fuente: Elaboración propia. 
Index, SPI, propuesto por T.B. Mc Kee y colaboradores en 1993, muy utilizado en estudios sobre sequías que emplean o no información satelital (Vicente-Serrano, et al., 2004; Vicente-Serrano, 2007; García et al., 2007; Jain et al., 2010).

\section{Consideraciones finales}

Los índices WDI y TVDI son de fácil implementación, una vez obtenidas las imágenes de NDVI, TS y TA necesarias para su aplicación. Logran reproducir diferencias espaciales y temporales suficientemente claras, incluso por observación directa de las imágenes resultantes, por lo que se piensa que pueden convertirse en un método adecuado y accesible para estudiar potencial déficit hídrico en áreas de vegetación natural y agrícola-forestal, y hacer un seguimiento del fenómeno en periodos de distinta longitud. En este contexto, pueden derivarse avances significativos, no solo en la determinación de déficit hídrico o en un caso más extremo de sequía, sino que, consecuentemente, estos avances pueden influir en el diseño de políticas públicas para un mejor aprovechamiento del recurso agua de riego. Por otra parte, en el ámbito privado estos avances podrían colaborar en la focalización de los cultivos, según sus requerimientos hídricos en áreas de secano, donde se aprecian diferencias en la humedad del suelo de acuerdo a variables locales.

Dado el tamaño del área de estudio no existe una diferenciación climática significativa en la región, más allá de la que representan las variaciones altitudinales y la disposición de los cordones orográficos que son los que definen, precisamente, las condiciones de riego/secano de la región. Esta situación aparece como una variable que podría mejorar las estimaciones de humedad del suelo al tratarla como una variable específica. Sin embargo, aun asumiendo una homogeneidad climática en el área de trabajo, se requiere todavía de estudios de validación en cuanto a la precisión de los resultados obtenidos, ya que solo así se podrá contar con una herramienta confiable en ambientes como los mediterráneos, sujetos a episodios de variaciones considerables en las precipitaciones que pueden resultar extremos. Esto último, precisamente, ha ocurrido en Chile en Ios últimos veinte años donde se han presentado episodios de sequía en vastas zonas del país, las que podrían ser mitigadas con indicadores de alerta temprana basados en este tipo de estudio, tal como se plantean diversos autores en el mundo.

Las consideraciones que deben tenerse en cuenta para la implementación adecuada de estos métodos son de distinto tipo. Por una parte, la calidad de los datos de entrada es esencial, en particular, en la interpolación de la temperatura del aire en caso del WDI, cuya exactitud dependerá tanto de los valores de campo con que se cuenta (estaciones meteorológicas) y, muy especialmente, en el algoritmo de interpolación, cuestión particularmente compleja cuando existen modificadores de la termodinámica atmosférica, como la orografía y la distancia a grandes masas de agua. Este es un punto que requiere mayor profundización y que se espera acometer. Otra cuestión de gran relevancia es la comprensión conceptual del significado tanto del trapezoide como del triángulo que definen los límites húmedo y seco de los métodos WDI y TVDI, respectivamente. Variaciones pequeñas en las pendientes de estos trazos resultan en diferencias significativas de las estimaciones de humedad del suelo.

Finalmente, debe considerarse que el cálculo de estos métodos para la obtención de mapas o imágenes de humedad/sequedad no están implementados como herramientas en los sistemas de información geográfica actuales, por lo que aún deben desarrollarse los procedimientos manualmente o codificarlos si el sistema lo permite, lo que resulta bastante laborioso en cualquier caso.

\section{Referencias bibliográficas}

ANDERSON, K. Software for processing HDF-EOS data User's Guide Version 1.8 (2005). Helsinki: VTT Information Technology, 2005. Disponible en internet: http://www. cr.chiba-.jp/databases/modis/modis_win/ hdfeos_guide.pdf

CARVACHO, L. y SÁNCHEZ, M. Espacialización de la evapotranspiración potencial, ETP, con imágenes NOAA-AVHRR, en la 
VI Región del Libertador General Bernardo O’Higgins. Anales de la Sociedad Chilena de Ciencias Geográficas, 2008, p. 31-34.

CHOUDHURY, B.J. Synergism of multispectral satellite observations for estimating regional land surface evaporation. Remote Sensing of Environment, 1994, Vol. 49, p. 264-274.

CHUVIECO, E. Teledetección ambiental. La observación de la Tierra desde el espacio. Barcelona: Editorial Ariel, Nueva edición actualizada, 2010.

DESBOIS, N.; PEREIRA, J.M.; BEAU DOIN, A.; CHUVIECO, E. \& VIDAL, A. Short term fire risk mapping using remote sensing. In: CHUVIECO, E. (editor). A review of remote sensing methods for the study of large wildland fires (MEGAFiReS Project ENVCT96-0256). Alcalá de Henares: Universidad de Alcalá, 1997, p. 29-60.

DIRECCIÓN METEOROLÓGICA DE CHILE. Zonificación Agroclimática VI Región. Santiago de Chile, 2003.

FENSHOLT, R. \& SANDHOLT, I. Derivation of a shortwave infrared water stress index from MODIS near-and shortwave infrared data in a semiarid environment. Remote Sensing of Environment, 2003, Vol. 87, p. 111121.

GARCíA, S.; BAILLE, A.; GONZÁLEZ, M.M.; MARTÍNEZ, V.; URREA, M.; HERNÁNDEZ, Z.; NORTES, P. y TANGUY, M. Desarrollo y aplicación de indicadores de alerta temprana frente a sequías a escala regional desde MODIS. En: RIVAS, R.; GRISOTTO, A. y SACIDO, M. (editores). Teledetección - Hacia un mejor entendimiento de la dinámica global y regional. Buenos Aires: Ed. Martin, 2007, p. 223-229.

GILLIES, R.R.; CARLSON, T.N.; CUI, J.; KUSTAS, W.P. \& HUMES, K.S. A verification of the "triangle" method for obtaining surface soil water content and energy fluxes from remote measurements of the Normalized Difference Vegetation Index (NDVI) and surface radiant temperature. International Journal of Remote Sensing, 1997, Vol. 18, № 15, p. 3145-3166.
GOETZ, S.J. Multi-sensor analysis of NDVI, surface temperature and biophysical variables at mixed grassland site. International Journal of Remote Sensing, 1997, Vol. 18, № 1, p. 71-94.

GUPTA, R.K.; PRASAD, S.; SESHA SAI, M.U.R. \& VESWANAGHAM, T.S. The estimation of surface temperature over an agricultural area in the State of Haryana and Panjab, India, and its relationship with the Normalized Difference Vegetation Index (NDVI), using NOAA-AVHRR data. International Journal of Remote Sensing, 1997, Vol. 18, No 18, p. 3729-3741.

JAIN, S.K.; KESHRI, R.; GOSWAMI, A. \& SARKAR, A. Application of meteorological and vegetation indices for evaluation of drought impact: a case study for Rajasthan, India. Natural Hazards, 2010, No 54, p. 643-656.

KALLURI, S.N.V.; TOWNSHEND, J.R.G. \& DORAISWAMY, P. A simple single layer model to estimate transpiration from vegetation using multi-spectral and meteorological data. International Journal of Remote Sensing, 1998, Vol. 19, No 6, p. 1037-1053.

KUSTAS, W.P. \& NORMAN, J.M. Use of remote sensing for evapotranspiration monitoring over land surfaces. Hydrological Sciences Journal, 1996, Vol. 41, № 4, p. 495-517.

LÓPEZ, R.; ARTEAGA, R.; VÁSQUEZ, M.; LÓPEZ, I. y SÁNCHEZ, I. Índice de estrés hídrico como un indicador del momento de riego en cultivos agrícolas. Agricultura Técnica en México, 2009, Vol. 35, № 1, 97-111.

MORAN, M.; CLARKE, T.R.; INOUE, Y. \& VIDAL, A. Estimating crop water deficit using the relation between surface-air temperature and spectral vegetation index. Remote Sensing of Environment, 1994, № 49, p. 246263.

MORAN, M.; RAHMAN, A.; WASHBURNE, J.; GOODRICH, D.; WELTZ, M. \& KUSTAS, W. Combining the Penman-Monteith equation with measurements of surface temperature and reflectance to estimate evaporation rates of semiarid grassland. Agricultural 
and Forest Meteorology, 1996, No 80, p. $87-$ 109.

NEMANI, R.R. \& RUNNING, S.W. Estimation of regional surface resistance to evapotranspiration from NDVI and Thermal-IR AVHRR data. Journal of Applied Meteorology, 1989, Vol. 28, p. 276-284.

NEMANI, R.; PIERCE, L.; RUNNING, S. \& GOWARD, S. Developing satellite- derived estimates of surface moisture status. Journal of Applied Meteorology, 1993, Vol. 32, p. 548-557.

NOVOA, R. y VILLASECA, S. (editores). Mapa Agroclimático de Chile. Santiago de Chile: Instituto de Investigaciones Agropecuarias (INIA), 1989.

PERTOVT, L.E.; SCHIRMBECK, J.; RIVAS, R. y WÖHL COELHO, O.G. Determinación de stress hídrico de vegetación mediante imágenes de satélite AVHRR. In: Anais XIII Simpósio Brasileiro de Sensoriamento Remoto, Florianópolis, Brasil, 2007, p. 2.999-3.001.

PERTOVT, L.E.; WÖHL COELHO, O.G.; SCHIRMBECK, J. y RIVAS, R. Análisis de condicionantes ambientales del estrés hídrico de la vegetación en el sur de Brasil mediante imágenes NOAA-AVHRR. Boletín Geológico y Minero, 2008, Vol. 119, No 1, p. 119-124.

PRICE, J.C. Estimation of regional scale evapotranspiration trought analysis of satellite thermal infrared data. IEEE Transactions on Geoscience and Remote Sensing, 1982, Vol. GE-20, No3, p. 286-292.

PRICE, J.C. Using spatial context in sateIlite data to infer regional scale evapotranspiration. IEEE Transactions on Geoscience and Remote Sensing, 1990, Vol. 28, № 5, p. 940-948.

PROSPER-LAGET, V.; DOUGUÉDROIT, A. \& GUINOT, J.P. Mapping the risk of forest fire ocurrence using NOAA satellite information. EARSel Advances in Remote Sensing, 1995, Vol. 4 (3-XII), p. 30-38.

SÁNCHEZ, M. Modelos y aproximaciones para la estimación de la evapotranspiración con información satelital. Revista de Geografía Norte Grande, 2002, № 29, p. 107-120.

SÁNCHEZ, M. y CHUVIECO, E. Interpolación espacial de la evapotranspiración del cultivo de referencia, ETo, a partir de imágenes de satélite. Estudios Geográficos, 2002, LXIII, No 247, p. 261-277.

SÁNCHEZ, M. y CARVACHO, L. Estimación de evapotranspiración potencial, ETP, a partir de imágenes NOAA-AVHRR en la VI Región del Libertador General Bernardo O'Higgins. Revista de Geografía Norte Grande, 2006, № 36, p. 49-60.

SANDHOLT, I.; RASMUSSEN, K. \& ANDERSEN, J. A simple interpretation index space for assessment of surface moisture status. Remote Sensing of Environment, 2002, Vol. 79 , p. 213-224.

TUCKER, C. A spectral method for determining the percentage of green herbage material in clipped sample, Remote Sensing of. Environment, 1980, Vol. 9, p. 175-181.

U.S. WATER CONSERVATION LABORATORY. Thermal crop water stress index. Phoenix, Az, 2001. Disponible en internet: http://www.plantstress.com/articles/drought_i/ drought_i_files/CWSI_phoenix.pdf

VÁZQUEZ, P.; RIVAS, R. y USUNOFF, E. Análisis multitemporal de la disponibilidad de agua en el suelo a partir de datos de satélite. Ciencia, 2008, Vol. 3, No 3, p. 47-58.

VICENTE-SERRANO, S.M.; PONS-FERNÁNDEZ, X. \& CUADRAT-PRATS, J.M. Mapping soil moisture in the central Ebro river valley (northeast Spain) with Landsat and NOAA satellite imagery: a comparison with meteorological data. International Journal of Remote Sensing, 2004, Vol. 25, No 20, p. 4325-4350.

VICENTE-SERRANO, S.M. Evaluating the impact of drought using remote sensing in a mediterranean, semi-arid region. Natural Hazards, 2007, No. 40, p. 173-208.

VIDAL, A. \& DEVAUX-ROS, C. Evaluating forest fire hazard with Landsat TM derived water stress index. Agricultural and Forest Meteorology, 1995, Vol. 77, p. 207-224. 
WAN, Z.; WANG, P. \& LI, X. Using MODIS Land Surface Temperature and Normalized Difference Vegetation Index products for monitoring drought in the southern Great Plains, USA. International Journal of Remote Sensing, 2004, Vol. 25, № 1, p. 61-72.

WANG, P.X.; LI, X.; GONG, J. \& SONG, C. Vegetation temperature condition index and its application for drought monitoring. In: Proceedings of International Geoscience ans Remote Sensing Symposium, Sidney, 2001, p. 141-143.
XIN, J.; TIAN, G.; LIU, Q. \& CHEN, L. Combining vegetation index and remotely sensed temperature for estimation of soil moisture in China. International Journal of Remote Sensing, 2006, Vol. 27, $\mathrm{N}^{\circ} 10$, p. 2071-2075.

YANG, X.; ZHOU, Q. \& MELVILLE, M. Estimating local sugarcane evapotranspiration using Landsat TM image and a VITT concept. International Journal of Remote Sensing, 1997, Vol. 18, № 2, p. 453-459. 